\title{
Mapping Convection using Pulsating White Dwarf Stars
}

\author{
M. H. Montgomery ${ }^{1,2}$ \\ ${ }^{1}$ Department of Astronomy, University of Texas, Austin, TX 78712, USA \\ ${ }^{2}$ Delaware Asteroseismic Research Center, Mt. Cuba Obs., Newark, DE, USA
}

\section{Parametrization of Convection Zone}

As shown by Montgomery (2005), the non-sinusoidal shape of the light curves of pulsating white dwarf stars can be used to constrain models of convection in these objects. In particular, $\tau$, the timescale on which the convection zone responds to a change in input flux at its base, can be parametrized as

$$
\tau=\tau_{0}\left(\frac{T_{\text {eff }}}{T_{\text {effo }}}\right)^{-N}
$$

where $\tau_{0}$ and $T_{\text {effo }}$ are the equilibrium values of $\tau$ and the effective temperature, respectively, $T_{\text {eff }}$ is the instantaneous effective temperature, and $N$ is an exponent which determines how rapidly the depth of the convection zone changes with $T_{\text {eff }}$.

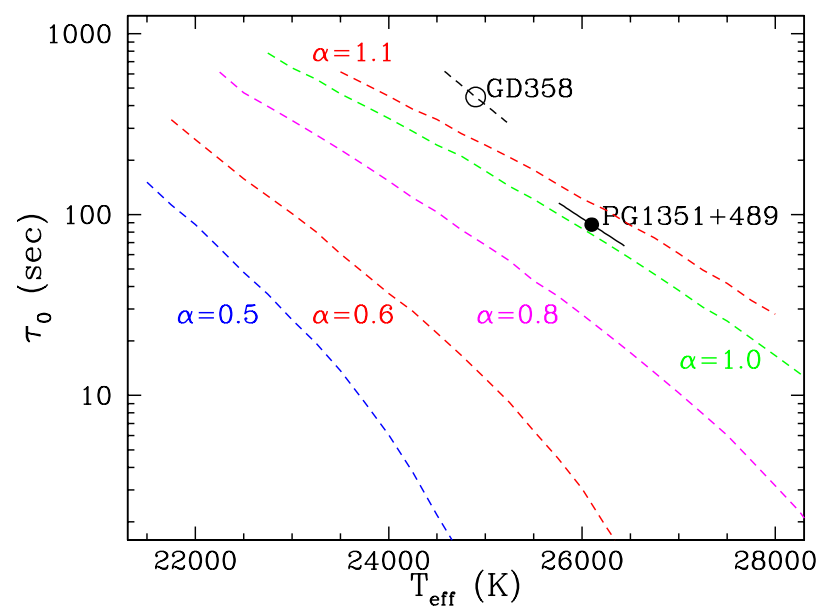

Figure 1: $\tau_{0}$ versus $T_{\text {eff }}$ assuming the pure $\mathrm{He}($ no $\mathrm{H}) T_{\text {eff }}$ values from Table 1.

Table 1: Derived convective parameters for two DBVs

\begin{tabular}{cccccc}
\hline star & $\theta_{\mathrm{i}}(\mathrm{deg})$ & $\tau_{0}(\mathrm{sec})$ & $N$ & $T_{\text {eff }}($ no H) & $T_{\text {eff }}($ with H) \\
\hline GD 358 & 62 & 450 & 25 & $24900 \mathrm{~K}$ & $24700 \mathrm{~K}$ \\
PG1351+489 & 58 & 87 & 21 & $26100 \mathrm{~K}$ & $22600 \mathrm{~K}$ \\
\hline
\end{tabular}




\section{Mapping the DBV Instability Strip}

We currently have examined two stars in the DBV instability strip: PG 1351+489 and GD 358. In Table 1, we list the convective parameters of the fits to these stars, as well as the derived inclination angles, $\theta_{\mathrm{i}}$. In addition, we list the effective temperatures determined from spectroscopic fits (Beauchamp et al. 1999), both for the case of pure He atmospheres and for the case of $\mathrm{H}$ contamination.

In Fig. 1, we show the location and slopes of these stars in the $\log \tau_{0}-T_{\text {eff }}$ plane, and we show the predictions of the Böhm \& Cassinelli (1971) mixing length theory (ML2) for various values of $\alpha$ (dashed curves). ML2/ $\alpha=1.1$ provides a reasonable fit to the $\tau_{0}$ of these stars. We note that if the effective temperatures assuming $\mathrm{H}$ contamination are used, we obtain the nonsensical result that the cooler star has the thinner convection zone (i.e., smaller value of $\left.\tau_{0}\right)$, something which is not possible based on very general arguments.

Acknowledgments. This research was supported in part through National Science Foundation grant AST-0507639.

\section{References}

Beauchamp A., Wesemael F., Bergeron P., et al., 1999, ApJ, 516, 887

Böhm K.-H., Cassinelli J., 1971, A\&A, 12, 21

Montgomery M. H., 2005, ApJ, 633, 1142

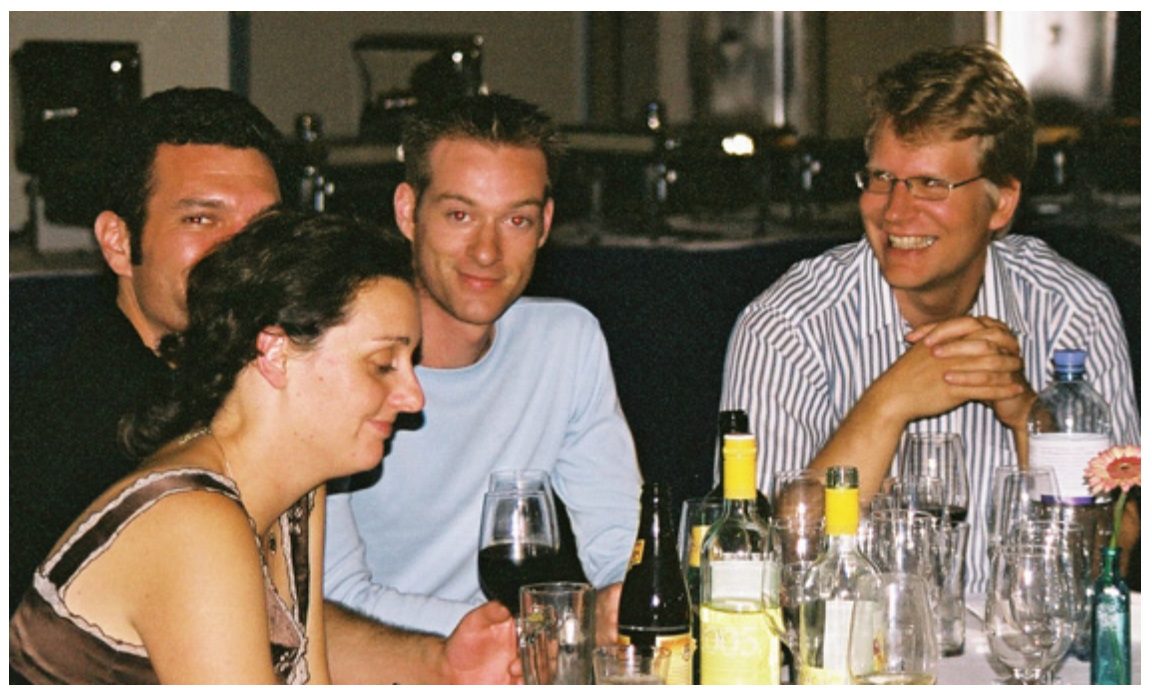

Orlagh Creevey, Travis Metcalfe (partly obscured), Dennis Stello and Mike Montgomery. 